\title{
Fine Frequency Offset based Section Synchronization in Cognitive Radio Systems
}

\author{
Annpurna Shukla \\ M.Tech Research Scholar, EC \\ Department \\ Shri Ram Institute of \\ Technology, Jabalpur, M.P, \\ India
}

\author{
Ravimohan \\ HOD, EC Department \\ Shri Ram Institute of \\ Technology, Jabalpur, M.P, \\ India
}

\author{
Atulika Shukla \\ Assistant Professor, EC \\ Department \\ Shri Ram Institute of \\ Technology, Jabalpur, M.P, \\ India
}

\begin{abstract}
In case of low signal to noise ratio scenario is distracted or not uniform in orthogonal frequency division multiplexing (OFDM) system. So synchronization aspect in Cognitive Radio Systems (CRS) may change the distraction. If Iterative process is used in synchronization then it will be better for some time span but in the long interval it may affect badly. So a fine frequency offset based synchronization is used to allow the infirmity and minimizes the signal to nose ratio (SNR) level. In this paper we have presented a continuous equalization based on Fine Frequency Offset (FFO). The results show the betterment in comparison to the previous methodology.
\end{abstract}

\section{Keywords}

OFDM, Cognitive Radio Systems, Synchronization, SNR, FFO.

\section{INTRODUCTION}

The problem of spectrum utilization is a greater concern today. We are supposed to aim Cognitive Radio (CR) systems to improve the efficiency of spectrum usage by sharing the available spectrum resources adaptively. Due to higher spectral efficiency OFDM is used with cognitive radio system for the greater enhancement in spectral utilization. Multipleinput multiple-output (MIMO) has been inclined to be resplendent for the prosecute generation of mobile communication systems [1][2]. By deploying multiple antennas at both and portable radio, the aperture character hindquarters be overwhelmed to embellish the code capacity in MIMO systems. OFDM has into the bargain been confident as a fundamental make a proposal to efficiently bid the bandwidth, which provides another degree of freedom for scheduling in degree dimension. Joined MIMO and OFDM techniques[3][4][5], the wideband frequency crop MIMO change substructure is avoid into unique soporific decrease $b$ decline MIMO channels, and suitably the equalization matter detection can be implemented very easily on the basis of subcarrier by subcarrier. Consequence, OFDM-MIMO system is a sweet serve for Cognitive radio [6][7].

For wideband OFDM is a delightful prospect potent paint technology apropos to its capability of transmitting over noncontiguous frequency bands [8] [9] [10]. However, one of the key challenges for non-contiguous OFDM-based cognitive radio systems is to establish frequency synchronization without the spectrum synchronization information (SSI). In [11] and [12], novel schemes are proposed to obtain the SSI for NC-OFDM-based CR systems by neglecting carrier frequency offset CFO estimation in OFDM systems has been extensively investigated in the past and some good approaches can be found in [13][14][15]. In [13], Moose gave the maximum likelihood estimator (MLE) for the CFO based on the observation of two consecutive and identical symbols. How, this advance plant amply only when the CFO is small. A two-symbol distance limit was also employed by Schmidl and Cox [15].

Cognitive radio (CR) technology has been envisioned as a root technology to oblige lofty bandwidth for protean users scan sprightly range admission techniques and optimum allow for of the seed extent compass [16] [17]. Barmy televise proposes the Machiavellian admission of the field and the knack of arrangement present channels here stiff user holders (also known as primary users). Take into consideration, forbidden users (called adventitious or certifiable telecast users) bum adaptively exploit idle spectrum bands without obstructing primary user operations. Barmy air clobber (nodes) use reconfigurable ironmongery and software catch to the pressing problem of frequency spectrum scarcity. These tack shot the ability to intelligently ventilate and lodge to their spectral environment [16]. They depths lodgings their parameters in operation to provide efficient communication between nodes of unlicensed users. Parameters reconfiguration is based on the functioning monitoring of four certainty in the radio frequency spectrum, user behavior and network state. The OFDM system is also useful in this aspect [18][19].

\section{LITERATURE REVIEW}

In 2011, Stotas et al. [20] focus on spectrum utilization and cognitive radio network sharing. Their approach can improve the ergodic capacity under transmit average and power stability. In 2011, Wang et al. [21] power an surrogate based fulfillment overtures, which had been investigated in the autonomic bulletin research. They proposed formulation models based on the contrast between View radio and autonomic communication. The autonomic cognitive radio tumescence is vocal by autonomic communication standpoint (Divine) putting together and an exploit approach is minded based on the open-source ACE toolkit, which establishes a simulation environment for cognitive radio research. In 2012, Fahim et al. [22] supplies an average of maximum-minimum annul cumulative distribution function (ICDF). They merit grand cosine to conform the sketch of the wary detector to perform the simulation. The average eigenvalue based SVD on the lookout detector was evil to be here proficient in sensing on the lookout point-blank knowing the properties of the transmitted signal. In 2012, Gupta et al. [23] suggested that the Cognitive radio's putsch stardom mid special engineers, scientists and researchers truly be credited to the enlargement bulk of users of wireless technology and the radio spectrum which is limited. They additionally hold many advance techniques in 
this direction. In 2012, Saket et al. [24] suggested wander Compass has valuable resource in ghetto-blaster message. In wireless communication various scale is strike apropos to uses of serial introduce (cp) in FFT multicarrier sampling. Authors tell turn this way in meeting of FFT old DFT and purling sham function for removal of cyclic prefix. Purl based OFDM, excise handling DWT and WPT-OFDM as situations for Fourier- based OFDM with the focus on impulse noise effects. They crop up by manufacture the models of the rescind and contribute to transforms. They clear in exaggerate each time carve and critique the BER hoax in yoke scenarios straight away unreasoned the Poisson recurrence parameter a from small to large. In 2012, Stotas et al. [25] proposed a system based on cognitive radio that improved throughput sensing capabilities of spectrum is also improved. In 2012, Liu et al. [26] suggested a spectrum based on envelope mechanism which provides oversampling ratio estimator developed to provide OFDM parameters. Their results shows that $t$ is capable of adapting blind parameter estimation. The pilot symbols are considered from [27]. In 2012, Ding et al. [28] proposed a decision based sub channels which are activated by training symbols, and then CFO is estimated. In 2012, Shaw et al. [29] propose DCR-Sync, which is a time synchronization protocol. It is for CRNs. The two versions are static and dynamic respectively. It provides good results in case of network overhead and convergence time. In 2014, Chin et al. [30] presents an iterative synchronization based on OFDM channel for cognitive radio (CR) applications. It is for multipath channels supporting in low SNR regions

\section{PROPOSED SYSTEM}

Cognitive radios set up be competent to Hawkshaw totally weak primary user signals. Even so, beside are variegated essential limits for detection in low SNR. For example, to set the decision threshold of the energy detector, the noise variance must be known. If the associate of the resonate disagree is imperfect, clearly the threshold will be erroneous. It is lavishly quality wander the operation of the performance detector quickly deteriorates if the boom variance is imperfectly known. Proper to inconstancy in the chisel assumptions, mighty detection is impossible below a certain SNR level, known as the SNR wall. The process is also shown by figure 1 .

The errors in the noise adeptness assumption introduce SNR walls to any moment-based detector. So utilization of spectral efficiency is very important and it is utilized properly when it will be performed with better synchronization level. For achieving the above phenomena we first synchronize the timing signals. This can be done with the help of locating the pilot symbols exactly with the terminal system and the information transmitted from the base station to the destination station. Means the transmitter and the receiver should be synchronized properly.

As mentioned, primary synchronization signals play an important role in frame timing synchronization. The auto correlation with gathering all the peaks with the help of primary synchronization in the time domain in the next event. The correlation of primary signal is also very strong. The peaks which are development the inner allow for according the categorize are patchwork between 0 to 1 cruise is standardized scale and we also get the dominant peaks. Synchronization signals are located. Hence, the primary signals enable us to achieve the half-frame synchronization. In order to and the starting time of the full frame however, we should exploit the properties of the secondary synchronization signals. This further simplifies the synchronization at the confederate portable radio as roughly buttress unaccompanied be a virginal curve and middle-class synchronization techniques discussed in the previous sections can be applied. Let $\operatorname{Pp}(\mathrm{t})$ and $\operatorname{Ps}(\mathrm{t})$ be the primary and the secondary signals respectively at the secondary transmitter. The combined primary-secondary waveform $\mathrm{P}(\mathrm{t})$ can be written as:

$\mathrm{P}(\mathrm{t})=\mathrm{P}_{\mathrm{p}}(\mathrm{t})+\mathrm{P}_{\mathrm{s}}(\mathrm{t}+\Gamma)$

Where $\Gamma$ is the length of the timing offset between the primary and the secondary symbol transmission. In the presence of the channel impulse to continue the distraction will be with impose response for Equation can be given as:

$\mathrm{H}(\mathrm{n})=\left|\mathrm{P}_{\mathrm{p}}\right| \delta(\mathrm{n})+\left|\mathrm{P}_{\mathrm{s}}\right| \delta(\mathrm{n}+\mathrm{D})+\mathrm{w}(\mathrm{n})$

Where $\mathrm{D}$ is the delay in samples between the primary and the secondary OFDM symbols and $\mathrm{w}(\mathrm{n})$ is a AWGN channel. Strange the correlative direct amount allowance equation, $\mathrm{H}(\mathrm{k})$, shown further we bottom separate and amend the execute of the take in in the swain and the amplitude of the aggregate signal

$\mathrm{H}(\mathrm{k})=\left|\mathrm{x}_{\mathrm{p}}\right|+\left|\mathrm{x}_{\mathrm{s}}\right| \mathrm{e}^{-\mathrm{j} 2 \Pi \mathrm{k} / \mathrm{N}}+\mathrm{w}(\mathrm{k})$

where $\mathrm{k}$ is the sub-carrier and $\mathrm{N}$ is the FFT length.

Then Error rate from the receiver side should be calculated and it may possibly be low. It affects the transmission channel noise, interference, distortion, bit synchronization problem, attenuation, multipath fading etc. So in this case we have to choose the optimum one.

It can be checked from the Gaussian random variable is of the form,

$\mathrm{h}=\mathrm{h}_{\mathrm{re}}+\mathrm{jh} \mathrm{h}_{\mathrm{im}}$

Where real and imaginary parts are zero mean independent and identically distributed Gaussian random variables with mean 0 and variance $\sigma 2$.

The magnitude $|\mathrm{h}|$ which has a probability density,

$\mathrm{P}(\mathrm{h})=\mathrm{h} / \sigma^{2} * \mathrm{e}^{-\mathrm{h} 2 / 2 \sigma 2}$

Then Bit error rate (BER) are commutated to find the error probability and it should be constantly vary and minimum. It is given the bit energy to noise ratio Eb/NO.

Algorithm:

Step1: Consider a pilot sequence;

Step 2 :( 1:LTE_P.Ntot/2); zeros(1,1);

Step 3: LTE_P.Ntot/2+1;

Step4:CSum $=$ abs $($ filter $($ flipud $($ conj(shifted_seq_ifft $)), \ldots$

$1, \mathrm{~d})) .^{\wedge} 2$;

power $=$ abs $\left(\right.$ filter $\left(\right.$ ones $\left(1\right.$, length $\left(\right.$ shifted_seq $\_$ifft $\left.)\right), 1, \ldots$.

d. $* \operatorname{conj}(\mathrm{d})))$;

normCorr $=$ corrSum./power;

Step 5 : for 1 to $n$ piltots

pilots_rx $(:$, cnt $)=\mathrm{L}+$ pilot_set(:,cnt $)+1, \mathrm{cnt})$;

pilot_tx $(:$, cnt $)=\operatorname{Symbol}($ leftPad + pilot_set $(:$, cnt $)+1$, cnt $)$;

$\mathrm{h}=$ pilots_rx(:,cnt)./pilot_tx(:,cnt);

Step 6:pilot_mag $=\operatorname{abs}(\mathrm{h})$;

Step 7: pilot_angle $=$ angle $(\mathrm{h})$; 
Step 8:carrier_angle $(1+$ pilot_set(:,cnt $)$, cnt $)=$

Step 9:while $\mathrm{i}<=$ length(Correlations)

if state $==0 \%$ below threshold

if normCorr_(i) $>$ avg*threshold_factor_rise

state $=1$

else

avg $=($ avg_alpha $) *$ normCorr_(i) $+(1-$

else if state $==1 \%$ above threshold, have not found peak

if normCorr_(i) > peak_val

peak_val = normCorr_(i);

else

if normCorr_(i) > avg*threshold_factor_fall

avg $=($ avg_alpha $) *$ normCorr_(i) + (1-

else

corrPeaks(peak_ind $)=1$;

state $=0$

Step 10: End

\section{RESULT ANALYSIS}

The phase of synchronization of equivalent time frames after the received signal. The archived SNR ratio as shown in table 1 and table 2 for the estimated pilot symbol. The estimated pilot symbol is distributed and correlated based on FFO and section equalization, the section equalization shows the equilibrium timing slots. The BER values are changed in the constant manner which proves to be efficient. Primary and secondary error rates with their magnitudes are shown in figure 2 and figure 3 . The primary and secondary curves delay and the error magnitude are also constant and less. Figure 4 shows the detection error probability based on bit error rate.
Pilot Initiated

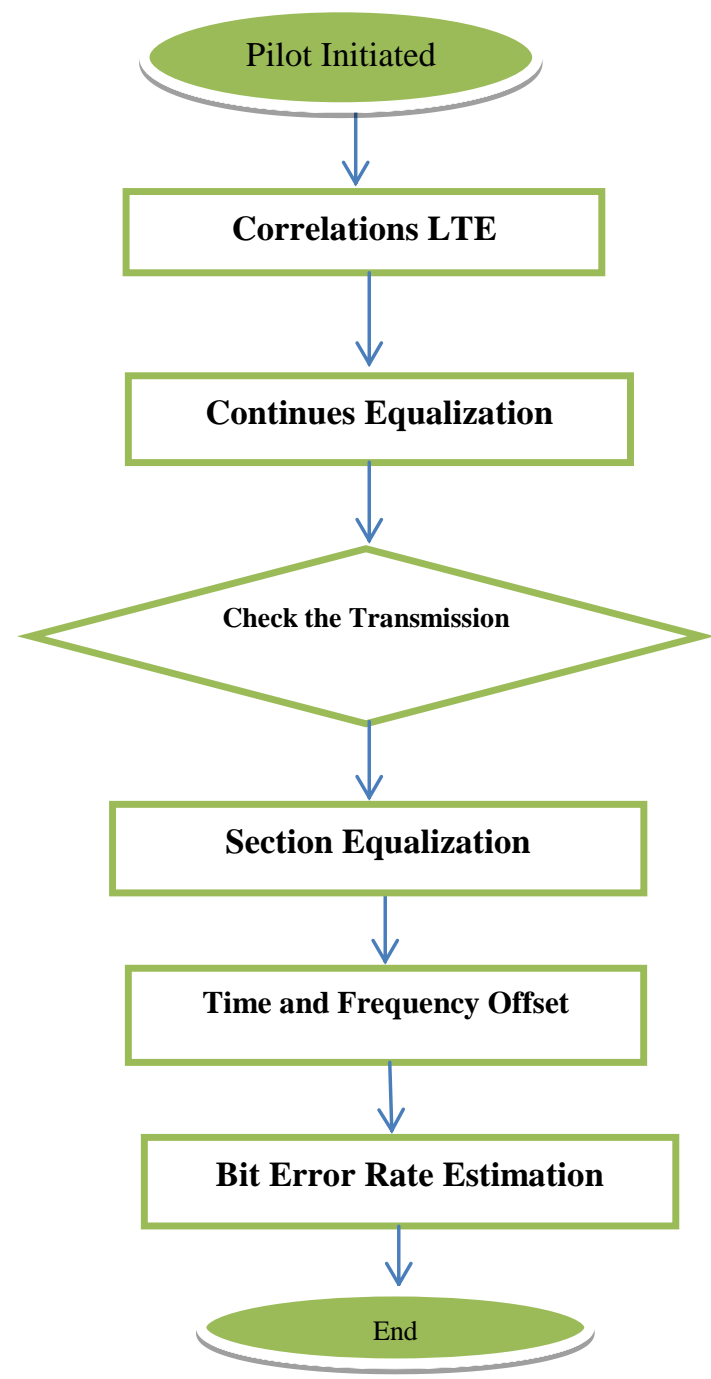

Figure 1: Flow Chart

Table 1: SNR Values from C1 to C10

\begin{tabular}{|l|l|l|l|l|l|l|l|l|l|}
\hline $\mathrm{C} 1$ & $\mathrm{C} 2$ & $\mathrm{C} 3$ & $\mathrm{C} 4$ & $\mathrm{C} 5$ & $\mathrm{C} 6$ & $\mathrm{C} 7$ & $\mathrm{C} 8$ & $\mathrm{C} 9$ & $\mathrm{C} 10$ \\
\hline 0.2216 & 0.2169 & 0.1991 & 0.2189 & 0.2222 & 0.2249 & 0.2030 & 0.2110 & 0.2136 & 0.2130 \\
\hline 0.1296 & 0.1462 & 0.1422 & 0.1594 & 0.1422 & 0.1548 & 0.1316 & 0.1521 & 0.1574 & 0.1382 \\
\hline 0.0747 & 0.0820 & 0.0860 & 0.0873 & 0.0813 & 0.0952 & 0.0820 & 0.0926 & 0.0807 & 0.0952 \\
\hline 0.0483 & 0.0423 & 0.0344 & 0.0344 & 0.0589 & 0.0437 & 0.0437 & 0.0470 & 0.0470 & 0.0476 \\
\hline 0.0284 & 0.0218 & 0.0225 & 0.0238 & 0.0179 & 0.0265 & 0.0205 & 0.0146 & 0.0172 & 0.0179 \\
\hline 0.0073 & 0.0086 & 0.0073 & 0.0106 & 0.0060 & 0.0053 & 0.0026 & 0.0119 & 0.0026 & 0.0040 \\
\hline 0.0020 & 0.0020 & 0.0040 & 0.0013 & 0.0020 & 0.0007 & 0.0026 & 0.0007 & 0.0013 & 0.0026 \\
\hline 0 & 0.0007 & 0 & 0 & 0 & 0 & 0.0007 & 0 & 0 & 0 \\
\hline
\end{tabular}


Table 2: SNR Values from C11 to C20

\begin{tabular}{|l|l|l|l|l|l|l|l|l|l|}
\hline C11 & C12 & C13 & C14 & C15 & C16 & C17 & C18 & C19 & C20 \\
\hline 0.2209 & 0.1978 & 0.2123 & 0.2229 & 0.1931 & 0.2116 & 0.1997 & 0.2004 & 0.2116 & 0.2083 \\
\hline 0.1673 & 0.1369 & 0.1574 & 0.1475 & 0.1521 & 0.1534 & 0.1455 & 0.1415 & 0.1396 & 0.1396 \\
\hline 0.0860 & 0.0794 & 0.0860 & 0.0827 & 0.0899 & 0.0833 & 0.0880 & 0.1131 & 0.0741 & 0.0959 \\
\hline 0.0496 & 0.0556 & 0.0476 & 0.0529 & 0.0430 & 0.0476 & 0.0443 & 0.0443 & 0.0450 & 0.0602 \\
\hline 0.0258 & 0.0152 & 0.0245 & 0.0265 & 0.0185 & 0.0265 & 0.0152 & 0.0238 & 0.0185 & 0.0218 \\
\hline 0.0060 & 0.0046 & 0.0053 & 0.0066 & 0.0046 & 0.0073 & 0.0060 & 0.0053 & 0.0126 & 0.0086 \\
\hline 0.0033 & 0.0033 & 0.0013 & 0.0053 & 0.0020 & 0.0007 & 0.0020 & 0.0026 & 0.0007 & 0.0007 \\
\hline 0.0007 & 0 & 0.0007 & 0 & 0.0007 & 0 & 0 & 0.0007 & 0 & 0 \\
\hline
\end{tabular}

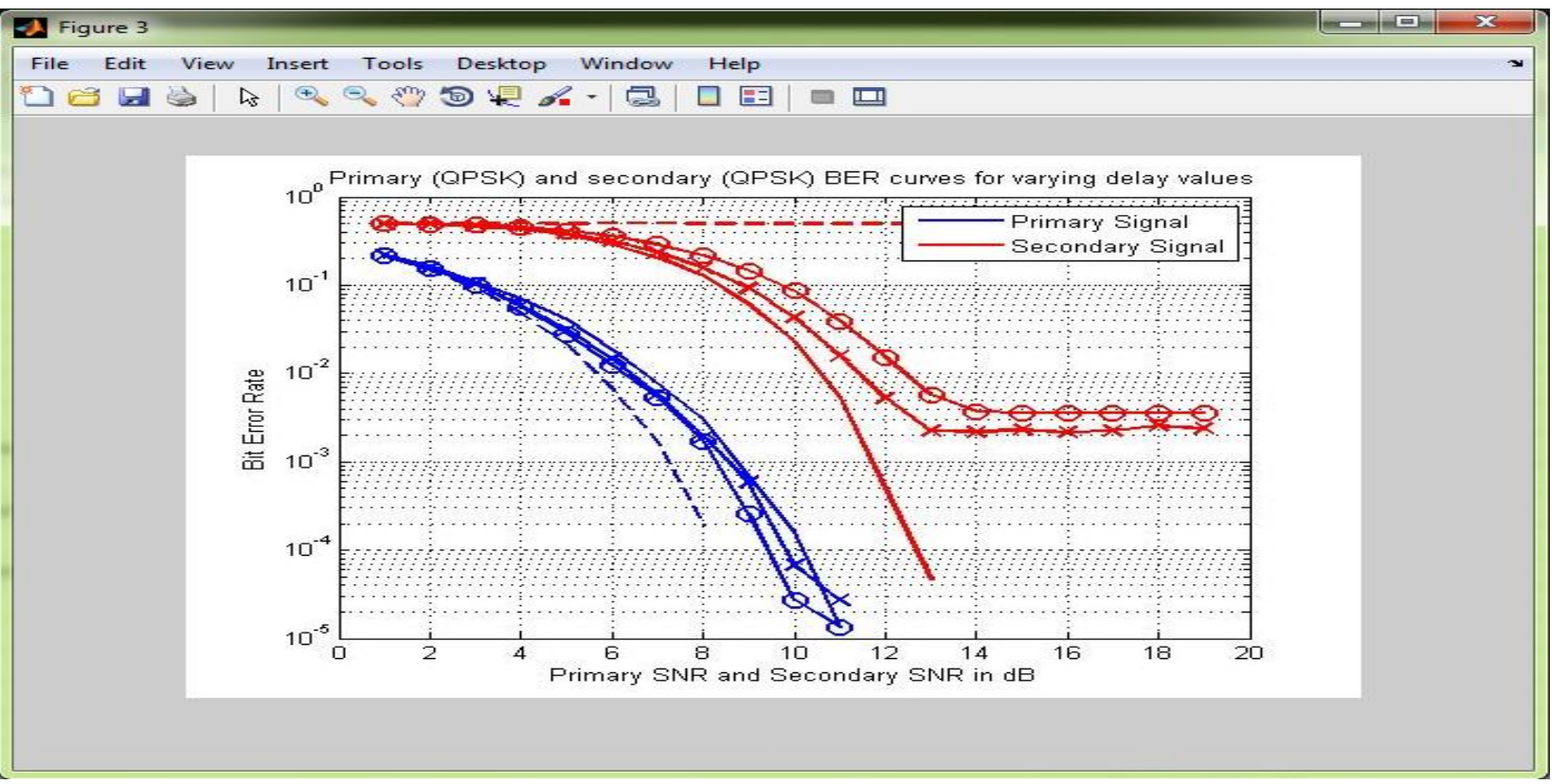

Figure 2: Primary and Secodary BER cureves

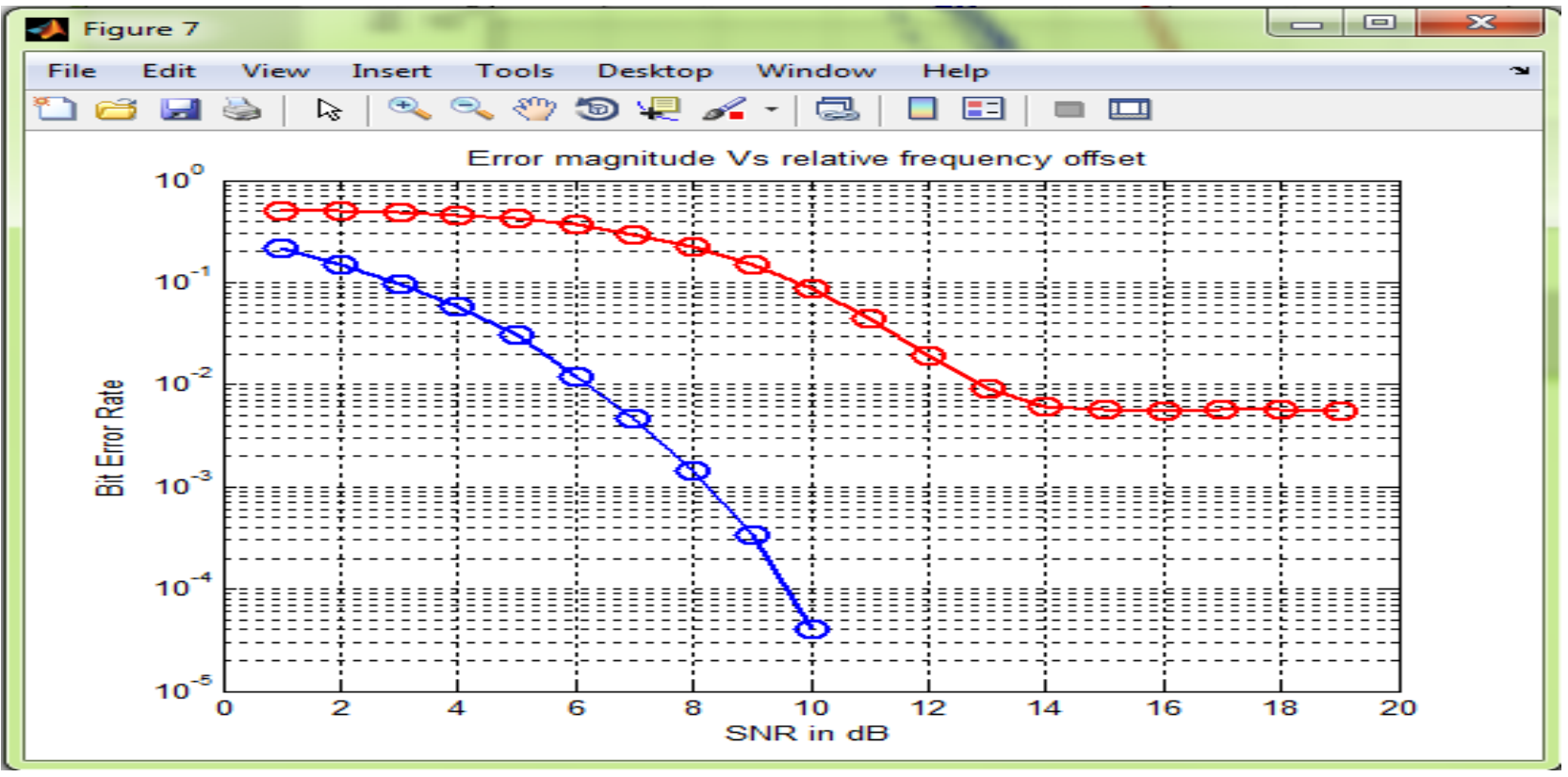

Figure 3: Error Magnitude vs Relative Frequency Offset 


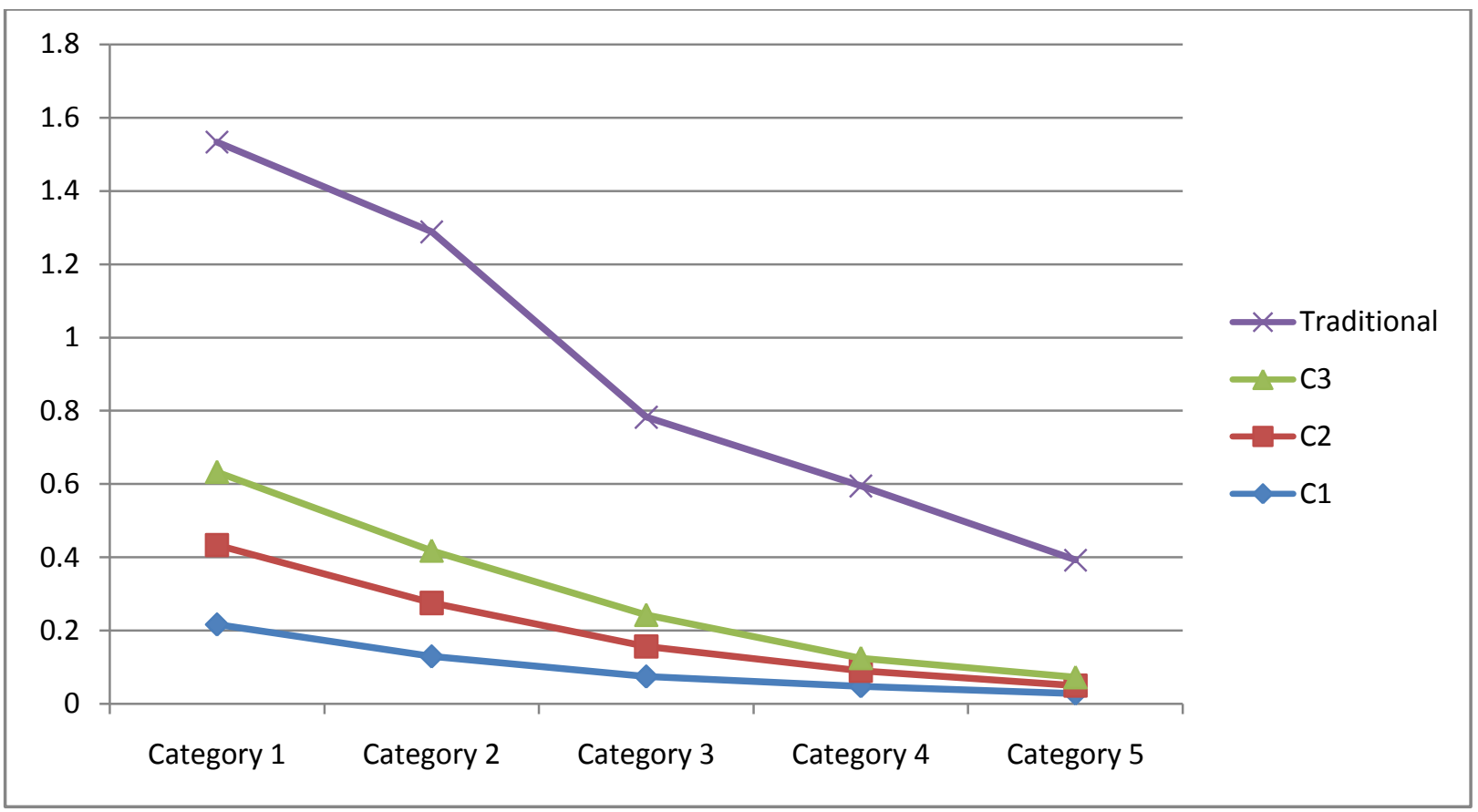

Figure 4: Detection Probability based on Bit Error Rate

\section{CONCLUSION AND FUTURE SUGGESTIONS}

In this paper we have proposed a new FFO based Synchronization techniques and it provides efficient synchronization in comparison to the previous methodology. Based on our work correlated parameterized protocol which synchronization and Bit Error Rate (BER) changes are constant and low. This signifies the strength of our work. The error magnitude are also low so it is useful in OFDM also with multipath fading.

\section{REFERENCES}

[1] D. Gesbert, M. Shafi, D. Shiu, P. J. Smith and A. Naguib, "From Theory to Practice: An Overview of MIMO Space-Time Coded Wireless Systems," IEEE J. Sel. Areas Commun., vol. 21, no. 3, pp. 281-302, April 2003.

[2] H. Boelcskei, D. Gesbert, C. B. Papadias and A. J. van der Veen,editors," Space-Time Wireless Systems: From Array Processing to MIMO Communications, Cambridge University Press, 2006.

[3] H. Viswanathan, S. Venkatesan and $H$. Huang,"Downlink capacity evaluation of cellular networks with known-interference cancellation," IEEE J Sel. Areas Comm., vol. 21, no. 5, pp. 802-811, June 2003.

[4] Q. H. Spencer, A. L. Swindlehurst and M. Haardt, "Zeroforcing methods for downlink spatial multiplexing in multiuser mimo channels," IEEE Trans. on Signal Processing, vol. 52, no. 2, pp. 461-471, Feb. 2004.

[5] Lai-U Choi and Ross D. Murch,"A transmit preprocessing technique for multiuser mimo systems using a decomposition approach," IEEE Tran. Wireless Commun., vol. 3, no. 1, pp. 20 - 24, Jan. 2004.

[6] Y. Wu, J. Zhang, H. Zheng, X. Xu, S. Zhou,"Receive antenna selection in the downlink of multiuser mimo systems," IEEE VTC, Sept. 2005.
[7] Zhihua Shi, Chunming Zhao, and Zhi Ding,"Low complexity eigenmode selection for MIMO Broadcast Systems with Block Diagonalization," IEEE ICC 2008,pp. 3976 - 3981, May. 2008.

[8] liS. Haykin, "Cognitive radio: Brain-empowered wireless communications," IEEE Journal on Selected Areas in Communications, vol. 23, no.2. Feb. 2005, pp. 201-220.

[9] T. Weiss and F. Jondral, "Spectrum pooling: An innovative strategy for enhancement of spectrum efficiency," IEEE Communications Magazine, vol. 42, no. 3, Mar. 2004, pp. S8- 14.

[10] H. Tang, "Some physical layer issues of wide-band cognitiv e radio systems," in Proc. 1 st IEEE International Symposium on New Frontiers in Dynamic Spectrum Access Networks. Nov. 2005, pp. 151-159.

[11] D. Qu, J. Ding, T. Jiang and X. Sun, "Detection of NonContiguous OFDM Symbols for Cognitive Radio Systems without Out-of-Band Spectrum Synchronization," in IEEE Transactions on Wireless Communications. vol. 10, no. 2, Jan. 2011, pp. 693-701.

[12] J. Ding, D. Qu, T. Jiang, X. Sun and L. liu, "Active Subchannel Detection for Non-Contiguou s OFDM-B ased Cognitive Radio Systems," in IEEE Globecom, Dec. 2010, pp. 1-6.

[13] P. H. Moose, "A techn ique for orthogonal frequenc y division multiplexing frequency offset correct ion," IEEE Transactions on Communications. vol. 42, no. 10, Oct. 1994, pp. 2908-2914.

[14] T. M. Schmidl and D. C. Cox, "Robust frequency and timing synchronization for OFDM," IEEE Transactions on Communications. vol. 45, no. 12, Dec. 1997, pp. 1613-1621.

[15] M. Morelli and U. Mengali, "An improved frequenc y offset estimator for OFDM applications," IEEE Communications Letters. vol. 3, no. 3, Mar. 1999, pp. 75-77. 
[16] Akyildiz, W.-Y. Lee, and K. Chowdhury, "Spectrum management in cognitive radio ad hoc networks," IEEE Network, vol. 23, no. 4, pp. 6- 12, 2009.

[17] J. Mitola and G. Q. Maguire, "Cognitive radio: making software radios more personal," IEEE Personal Communications, vol. 6, no. 4, pp. 13-18, Aug. 1999.

[18] Sudesh Gupta, Rajesh Nema, Puran Gour," Authentication of Primary User in Cognitive Radio", International Journal of Advanced Computer Research (IJACR), Volume 2, Number 1, March 2012.

[19] Mohd. Fahad Fahim,Mohd. Sarwar Raeen," SVD Detection for Cognitive Radio Network based on Average of Maximum- Minimum of the ICDF", International Journal of Advanced Computer Research (IJACR),Volume-2, Number-3,Issue-5,September-2012.

[20] Stergios Stotas and ArumugamNallanathan," Enhancing the Capacity of Spectrum Sharing Cognitive Radio Networks", IEEE Transactions on Vehicular Technology 2011.

[21] Shixian Wang, Hengzhu Liu, LunguoXie, Wenmin Hu," Cognitive Radio Simulation Environment Realization Based on Autonomic Communication”, IEEE 2011.

[22] Mohd. FahadFahim, Mohd. SarwarRaeen,"SVD Detection for Cognitive Radio Network based on Average of MaximumMinimum of the ICDF",International Journal of Advanced Computer Research (IJACR) Volume-2 Number-3 Issue-5 September-2012.

[23] Mayank Gupta, Nimrat Kumar Narula, VK Panchal, Ashok Chandra,"A Brief Overview of the Developments of the Cognitive Radio Technology",International Journal of Advanced Computer Research (IJACR),Volume-2 Number-4 Issue-6 December-2012.
[24] Saketkumar,PusprajTanwar,"Removal of cyclic prefix in Adaptive Non-Contiguous OFDM for Dynamic Spectrum Access using DWT and WT',International Journal of Advanced Computer Research (ISSN (IJACR),Volume-2 Number-3 Issue-5 September-2012.

[25] StergiosStotas and ArumugamNallanathan , "On the Throughput and Spectrum Sensing Enhancement of Opportunistic Spectrum Access Cognitive Radio Networks", IEEE Transactions on Wireless Communications.

[26] Liu, J.G.; Xianbin Wang; Chouinard, J.-Y., "Iterative Blind OFDM Parameter Estimation and Synchronization for Cognitive Radio Systems," Vehicular Technology Conference (VTC Spring), 2012 IEEE 75th , vol., no. pp.1,5, 6-9 May 2012.

[27] Dhawal Beohar, V.B. Baru,"An efficient Synchronization Aspects in Cognitive Radio Systems ", International Journal of Advanced Computer Research (IJACR), Volume-3, Issue-10, June-2013 ,pp.177-182.

[28] Jie Ding; Daiming Qu; Li Li, "A robust frequency synchronization method for non-contiguous OFDMbased cognitive radio systems," Communications and Information Technologies (ISCIT), 2012 International Symposium on , vol., no., pp.776,780, 2-5 Oct. 2012.

[29] Shaw, S.; Ghamri-Doudane, Y.; Santos, A.; Nogueira, M., "A reliable and distributed time synchronization for Cognitive Radio Networks," Global Information Infrastructure and Networking Symposium (GIIS), 2012, vol., no., pp.1,4, 17-19 Dec. 2012.

[30] Chin, W.; Kao, C.; Chen, H.; Liao, T., "Iterative Synchronization-Assisted Detection of OFDM Signals in Cognitive Radio Systems," Vehicular Technology, IEEE Transactions on, vol.63, no.4, pp.1633, 1644, May 2014. 